\title{
El texto: una noción problemática
}

\author{
"The text: a problematic notion
}

\begin{tabular}{l}
$\frac{\text { Juan Carlos González Vidal }}{\text { Iván Ávila González }}$ \\
\hline Universidad de Michigan, Estados Unidos \\
\hline
\end{tabular}

\section{Resumen}

En este artículo se ponen de relieve algunas dificultades que a lo largo del tiempo se han tenido para delimitar la noción de 'texto'. Su uso frecuente en los ámbitos de la crítica literaria, de la lingüística, de la semiótica... pudiera remitirnos a la idea de que existe una concepción homogénea. Sin embargo, lejos de esto, dentro de una misma disciplina se aprecian diferencias notables que forzosamente inciden en las investigaciones que toman el texto (o lo que cada una considera como tal) como la unidad de análisis fundamental. Así, nuestra pretensión es proponer, basándonos en concepciones anteriores, una redefinición que resulte lo suficientemente flexible para ser operativa en distintos campos del conocimiento y para que responda a las necesidades de la era de las nuevas tecnologías de la comunicación.

Palabras clave: Texto, mensaje, código, materia expresiva, unidad comunicacional.

\section{Abstract}

This article highlights some difficulties that over time have had in delimiting the notion of 'text'. Its frequent use in the fields of literary criticism, linguistics, semiotics ... could refer us to the idea that there is a homogeneous conception. However, far from this, within the same discipline there are notable differences that necessarily affect research that takes the text (or what each considers as such) as the fundamental unit of analysis. Thus, our intention is to propose, based on previous conceptions, a redefinition that is flexible enough to be operational in different fields of knowledge and to respond to the needs of the era of new communication technologies.

Keywords: text, message, code, expressive matter, communicational unit.

Cómo citar este artículo:

González Vidal, J. C. y Ávila González I. (2019). El texto: una noción problemática. Revista Amauta, 17(34), pp.17-26. DOl: http://dx.doi. org/10.15648/am.34.2019.3
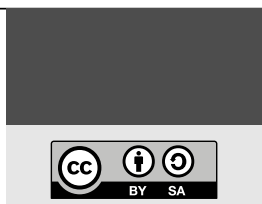

๑) 2019

\section{Correspondencia de autor:}

diac.umich@gmail.com avilaivan@umich.mx

\section{Recibido:}

5/Febrero/2019

Aceptado:

20/Mayo/2019

Publicado:

28/Junio/2019 


\section{Introducción}

La noción de 'texto' es una de las que más discusiones han suscitado en cuanto a su definición. A través del desarrollo de las ciencias del lenguaje y, más generalmente, de las disciplinas que se ocupan de la semiosis, se han observado dificultades de diversa índole para circunscribirla. En muchas ocasiones se parte de una generalización extrema, y se cae en el error de no ver las particularidades codiciales de los textos. Un caso es cuando se concibe un texto limitado a la escritura, o bien, cuando se lo enfoca como discurso. Es verdad que existen unidades textuales que se basan en la lengua como materia expresiva (en sus variantes oral y escrita), pero desde nuestra perspectiva, hay otras que no obedecen a esta conformación codicial.

Consecuentemente nos proponemos, entre otras cosas, rebasar esa postura lingüístico-dependiente y optar por una definición que sea operativa para el abordaje de textos de naturaleza diferente. Aquí se hace necesaria una aclaración. Es verdad que, con mucha frecuencia, y por la forma en que hablamos del 'texto', existiera una convención unánimemente reconocida, y que todo el mundo identificará la clase de conglomerado a la que se hace referencia con el término. Sin embargo, como se sabe, todavía no se elabora una definición que goce de tal reconocimiento. Vemos, hoy en día, concepciones diferentes que manifiestan entre sí aspectos coincidentes, pero igualmente, profundas divergencias e inconsistencias. Nuestra intención (y aquí radica el punto que deseamos aclarar) no es elaborar tal definición, sino reformular algunas que nos han antecedido para concretarlas en una que tenga mayor operatividad y así, en la medida de lo posible, evitar las problemáticas a que nos enfrentamos en este ámbito.

$\mathrm{Si}$, como afirmaba Lotman, todo texto es un mensaje (1978), forzosamente se compone de un plano de la expresión y de un plano del contenido; lo importante a destacar, es que el plano del contenido se organiza con base en la materialidad expresiva del mensaje.

Desde este instante podemos establecer una posición que nos permitirá lograr el objetivo planteado en el párrafo anterior: el respeto irrestricto a la especificidad codicial de cada género textual, por lo cual es preciso centrarse en una concepción que, de manera simultánea, tenga la flexibilidad suficiente para incluir una diver- 
sidad de unidades y, también, el rigor para ver rasgos genéricos de su composición estructural.

\section{La atención sobre el texto}

En fechas relativamente recientes, Oswald Ducrot y Jean-Marie Shaeffer afirmaban que, pese al empleo tan extendido de la noción de 'texto' en los dominios de la lingüística y los estudios literarios, no era frecuente encontrar una definición clara (1995, p.494). Esta falta de claridad, conjuntamente con la diversidad señalada precedentemente, han dificultado trabajar con una noción amplia que satisfaga las necesidades que plantean entidades basadas en soportes expresivos diferentes.

Las sociedades, conforme van desarrollándose, incrementan sus formas expresivas $y$, consecuentemente, sus potencialidades comunicativas y cognitivas. Este factor debe ser tenido muy en cuenta por la semiótica, puesto que, al estudiar la cultura como procesos significativo-comunicativos, requiere actualizar constantemente sus instrumentos analítico-descriptivos. En nuestra era, marcada por los avances tecnológicos, hemos asistido a una proliferación sin precedentes de formas expresivas en la mayor parte de las culturas conocidas. Piénsese, por ejemplo, en los videos musicales o en las redes sociales, que han dado lugar a la manifestación de entidades comunicativas que bien podrían considerarse como 'textos' desde una perspectiva adecuada.

Las ideas que expondremos están sujetas a desacuerdos, cosa que, lejos de rechazar, vemos positivamente. En los ámbitos científicos las confrontaciones de ideas contribuyen de manera determinante a su desarrollo'.

\section{El texto como entidad delimitada}

Recurriremos nuevamente a Ducrot y Shaeffer, que a mediados de la década de los noventa manifestaban lo siguiente:

\footnotetext{
$1 \quad$ Hace precisamente unos meses (a finales de enero de 2018) discutimos con algunos de nuestros colegas la pertinencia de aplicar la designación de 'texto' a un mural. Se enunciaron múltiples argumentos a favor y en contra. Aunque nos mantuvimos firmes en nuestra posición, extrajimos ideas que nos obligaron a reforzar aspectos que no tenían el suficiente sustento, lo que evidentemente redundará en este momento, cuando menos, en una mayor claridad expositiva.
} 
En accord avec l'usage répandu en pragmatique textuelle, on définira le texte ici comme une chaîne linguistique parlée ou écrite formant une unité communicationnelle $[\ldots](1995$, p.494).

De esta definición vamos a retener la idea de "unidad comunicacional", que nos permite de entrada establecer la noción de límite. Desde este punto de vista, para que un fenómeno semiótico sea considerado como texto, debe poseer una delimitación. Esta formulación resulta muy convincente, puede aplicarse a una amplia variedad de prácticas comunicativas. En refuerzo de lo anterior, Lotman y Cros (1978, 1983, respectivamente), entre otros, afirmaron que la noción de 'texto' activa inevitablemente el principio de inclusión/exclusión, es decir, que cada unidad de esta índole elige en el universo cultural los signos que entran en su constitución. La construcción textual comprende entonces, un proceso de selección que, de hecho, será la responsable de la producción de sentido.

El inconveniente que salta a la vista en la definición de Ducrot y de Shaeffer, es que restringe la materia expresiva al dominio lingüístico, postura que, por lo demás, ha gozado de una gran aceptación, cosa fácilmente constatable si recurrimos a las concepciones de algunos destacados investigadores. Por ejemplo, Louis Hjelmslev ve como texto la totalidad de una cadena lingüística, susceptible de analizarse de acuerdo al criterio de delimitación de las unidades mayores, que pueden ser la oración o el discurso, de modo que se dispondrá de perspectivas diferentes de análisis (Greimas y Courtés, 1982, pp.409-410). Aquí surge, además, el cuestionamiento relativo al instante en que un discurso se textualiza, en qué momento se dan esos límites.

Por su parte, Zellig Harris concibe el texto como un conglomerado de sintagmas entre los que operan relaciones de equivalencia sintáctica: para establecer dicha equivalencia, considera los elementos que pueden aparecer en un mismo contexto 0 , cuando menos, en un contexto similar. Además de la restricción contextual de la materia expresiva, está el hecho de que Harris deja de lado el aspecto semántico del conglomerado, es decir, que no considera las relaciones que a nivel del significado se establecen entre sus elementos sintácticos. Hoy en día, es evidente que en esta forma de proceder hay un error metodológico, porque toda entidad que se manifieste como texto tiene un nivel semántico que es precisamente el que le da el estatuto 
de unidad comunicacional (y cognitiva). Por otro lado, el lingüista norteamericano comparte con muchos de sus colegas la tendencia a trasladar casi mecánicamente modelos descriptivos de la oración al análisis textual. Inclusive, textos con soporte exclusivamente lingüístico, no pueden ser vistos como un conjunto de oraciones, pues estas aparecen cohesionadas por una estructura semántica subyacente.

Otro caso concreto en la línea argumentativa de Harris lo encontramos en Katz y Fodor (1963), quienes establecen que el texto se presenta como una megaoración, susceptible, consecuentemente, de dividirse en oraciones; las relaciones entre estas, en un marco textual serían equivalentes a las que propician las conjunciones al interior de las oraciones. Como se observa, el análisis se fundamenta, de nueva cuenta, en la lingüística de la oración y privilegia el nivel sintáctico.

Desde el momento en que la noción de texto se limita al ámbito lingüístico, es explicable que metodológicamente se realicen esta clase de translaciones.

Lo que debemos retener de las anteriores posturas, pese a las objeciones formuladas, es que implícitamente se halla comprendida la idea de límite textual: conjuntos de oraciones que se oponen a las oraciones no incluidas en ellos.

García-Berrío y Janos (1978) también piensan en el texto como una unidad lingüística, si bien su perspectiva es más amplia que la de aquellos que se limitan a utilizar los modelos de la lingüística de la oración. Ellos basan su reflexión sobre ciertos principios de la gramática generativa, y hablan, consecuentemente, de una estructura superficial y una estructura profunda (nivel semántico). Además, para la descripción de este último nivel, recurren a una lógica matemática, lo que les permite explicar las reglas que determinan la interacción entre ambos niveles. En síntesis, para ellos el texto es (...) un conjunto global de unidad comunicativa, un andamiaje lógico" (1978, p.56). Efectivamente, una unidad comunicativa puede ser considerada como tal solo en la medida en que comprenda una cohesión responsable, del tipo de vínculos que se establecen entre sus elementos o niveles constitutivos. Cualquier postura que omita el considerar el texto como una superposición sistemática de distintos niveles, limitará sus alcances descriptivos y analíticos. 
A pesar de los aciertos de García-Berrío y Janos, debemos nuevamente lamentar que sus reflexiones no se hagan extensivas a otro tipo de material expresivo².

Poco antes del fragmento citado de Ducrot y Shaeffer, ellos mismos habían hecho otra afirmación sobre la definición de texto:

[...] certains, enfin, lui donnent une extension trans-sémiotique, parlant de texte filmique, texte musical, etc. $(1995$, p.494)

Ellos consideran que designar como texto una unidad comunicacional construida con material expresivo distinto del lingüístico, es adoptar una postura trans-semiótica.

Por nuestra parte pensamos que, independientemente del fundamento teórico de tal afirmación, hay una falla evidente que va en dos sentidos y que podemos agrupar en los siguientes cuestionamientos: ¿acaso solamente los signos lingüísticos son expresiones semiósicas? y consecuentemente, ¿la semiótica no debe incluir en su campo de estudio otro tipo de expresiones, como las basadas en la graficidad, en la imagen en movimiento o en la música? En el primer sentido, que es más radical, los autores citados parecen negar el carácter semiótico de algunas expresiones sígnicas; en el segundo, todo indica que la posición adoptada, parte de los límites del campo de estudio de la disciplina, que excluiría -o debería excluir- otra clase de conglomerados sígnicos, a los que actualmente nadie les negaría el carácter de textos. Como se observa, la posición de Ducrot y Shaeffer implica una problemática de mayor envergadura, toda vez que tiende implícitamente a negar el aspecto diversificado y plural de las formas de comunicación humana.

De sobra sabemos que en cualquier cultura existen distintos tipos de actividad sígnica, y en este sentido nos vamos a permitir citar un argumento de Eco que hemos utilizado en otros trabajos:

Es difícil concebir un universo en que seres humanos comuniquen sin lenguaje verbal, limitándose a hacer gestos, mostrar objetos, emitir sonidos informes, bailar: pero igualmente difícil, es concebir en que los seres humanos sólo emitan palabras. (1977, p.263)

2 No debe pensarse que negamos a todos estos investigadores su derecho a delimitar su campo de estudio, simplemente pensamos que una consideración general de los fenómenos de textualidad hubiese permitido avances más significativos para describir estas formas de comunicación. 
Desde el punto de vista del semiotista italiano, todo en una cultura es susceptible de devenir una función semiótica, y no solamente eso, sino que, todo aquello con lo que una cultura tenga contacto tiene la posibilidad de traducirse en signos. Los función-signos de que hablaba Barthes (1985), que nosotros consideramos signos objetuales, representan un ejemplo contundente 3 . Un objeto, por su dimensión social, es portador de significados $y$, consecuentemente, implica una comunicación (al menos potencialmente). Que se piense en una simple lata de atún sobre una mesa: un observador puede conjeturar que alguien fue a comprar la despensa. En este mismo sentido, los fenómenos naturales también pueden convertirse en portadores de información convencionalizada: la forma y el color de las nubes, para quien sepa interpretar, indican la posibilidad de un clima frío, lluvioso; las olas grandes en el mar, peligro, etc.

Es precisamente por este motivo que Lotman (1996). concibe la cultura como un universo semiótico.

Desde esta perspectiva, no entendemos cómo pueden considerarse trans-semióticos (en cualquiera de los sentidos mencionados) fenómenos significativo-comunicativos como el cine o la música, dado que son actividades sígnicas altamente convencionalizadas que, además, obedecen a patrones culturales. Por supuesto que Ducrot y Shaeffer se refieren a textos y no a signos, pero como todo texto se halla contruido por materia sígnica, nuestra objeción continúa siendo válida.

Hemos tratado a los autores citados porque esas posturas, en ciertos círculos, continúan vigentes hasta nuestros dias. Una de las repercusiones más importantes de tal vigencia se da en las aulas de clase, donde en muchas ocasiones no se transmite una definición precisa de 'texto' y el alumno entra en confusión al enfrentarse a esta clase de unidades semióticas.

Hay que agregar, sin embargo, que en la actualidad observamos cierta apertura, ya que algunos teóricos apuestan por la diversidad. Por ejemplo, Wolgang Raible (2004), que aunque centra sus reflexiones en los textos lingüísticos (dada su formación), en el instante en que agrega el calificativo 'lingüísticos' implícitamente admite la existencia de otra clase de textos. Además, para explicar la jerarquización compo-

3 Una magnífica muestra de la utilización de signos objetuales la encontramos en la película Mon oncle, de Jacques Tati (1958), donde los artefactos connotan modernidad y actualidad. 
sitiva de una unidad textual, recurre a una analogía entre texto y la reproducción de una melodía: en ambos casos, se estará ante un conglomerado semántico cohesionado y funcional.

Con todo, esta apertura no se ha hecho extensiva con la rapidez deseada, pero lo destacable es que se está produciendo.

Así, es forzoso pensar en la existencia de textos de naturaleza expresiva diferente de la lengua, de modo que no solo es pertinente, sino necesario, hablar de textos pictóricos, literarios, cinematográficos, musicales, cartográficos, vestimentales, arquitectónicos... La comunicación, de la cual la producción textual forma parte, se constituye como un universo plurisistémico.

Ha pasado mucho tiempo desde la época(década de los sesenta) en que las investigaciones semióticas sobre cine, música, pintura, etc., adolecían de una sistematicidad conveniente para lograr acercamientos precisos. Actualmente para comprender mejor estos fenómenos, es posible tratarlos en calidad de textos.

\section{Por una definción operativa de texto}

Partiendo de lo expuesto anteriormente, proponemos la siguiente definción:

Se considerará 'texto' toda entidad comunicacional delimitada en sí misma y por sí misma, independientemente de su materia expresiva y de su extensión, que posea además una estructura semántica subyacente que cohesione sus elementos constitutivos.

Como se observa, lo que hemos hecho es ajustar varios de los enfoques citados para llegar a una concepción más operativa, la cual nos permite soslayar algunos problemas:

a. El discurso se convierte en texto en el momento en que se segmenta, en que se le ponen límites. Por ejemplo: al grabar una conversación.

b. Ciertas manifestaciones culturales, como rituales, danzas, cantos... desde el instante en que se presentan como conglomerados sígnicos con límites, es posible abordarlas en esta calidad. 
c. La cuestión relativa a intersección de entidades textuales de origen diferente (la intertextualidad) solo se explicará de manera pertienente si partimos de esta concepción flexible: canciones y pinturas en filmes 0 en novelas, poemas en novelas, relatos históricos en canciones...

Con respecto al último punto, nos gustaría citar un caso en el que se percibe con mucha claridad el acierto de partir de esta base teórica. Hace poco leímos Espejos en abismo. Estudios sobre la intertextualidad en la plástica, de Carmen V. Vidaurre. En él, la investigadora aborda aspectos de la producción pictórica de El Bosco, Durero y Goya, y nos revela de manera novedosa formas de generación de las obras seleccionadas con base en expresiones diferentes, evitando caer -como ella lo dice- en "[...] valoraciones meramente canónicas [...]" (2007, p.11). En el subtítulo mismo salta a la vista su postura teórico-metodológica.

A la vez, el planteamiento que elaboramos nos permite argumentar que un texto está dado de una vez y para siempre, en el sentido en que si hay variación en uno solo de sus elementos, ya no se tratará de la misma unidad. Inclusive, los llamados 'textos móviles', es decir, lo que puestos en un ordenador varían sus elementos periódicamente mediante un programa computacional, en el lapso de tiempo en que la unidad se mantiene estable, nos enfrentaremos a una entidad textual, con lo que la variación implicará otro texto.

Aquí debe quedar claro que nos referimos a las condiciones de producción (esas que determinan la construcción de un texto), no a las condiciones de recepción: la interpretación de una entidad textual varía de acuerdo a las circunstancias de recepción (Verón, 2004).

Como lo anunaciamos, nuestra intención no es proponer una concepción definitiva, sino contribuir a resolver ciertas problemáticas relacionadas con el término en cuestión. Dependerá del lector juzgar su pertinencia.

\section{Referencias bibliográficas}

Barthes, R. (1985). Sémantique de l'objet". En L'aventure Sémiologique. París, Francia: Seuil.

Cros, W. (1983). Literatura, Ideología y Sociedad. Madrid, España: Gredos. 
Ducrot, 0. \& Schaeffer, J. M. (1995). Nouveau Dictionnaire Encyclopédique des sciences du langage. París, Francia: Seuil.

Eco, U. (1977). Tratado de Semiótica General. Barcelona, España: Lumen.

García-Berrío, A. y Janos, S. P. (1978). Lingüística del texto y crítica literaria. Madrid, España: Alberto Corazón.

Greimas, A. J. y Courtés, J. (1982). Semiótica. Diccionario razonado de la teoría del lenguaje. Madrid, España: Gredos.

Katz, J. y Fodor, J. (1963). La estructura de una teoría semántica. México: Siglo XXI.

Lotman, I. (1978). Estructura del texto artístico. Madrid, España: Itsmo.

Lotman, I. (1996). La semiosfera. Semiótica de la cultura y del texto. Madrid, España: Cátedra-Universitat de València.

Raible, W. (2004). ¿Qué es un texto? Revista Función, (21-24). Guadalajara, México: Universidad de Guadalajara.

Verón, E. (2004). La Semiosis Social. Fragmentos de una teoría de la Discursividad. Barcelona, España: Gedisa.

Vidaurre, C. V. (2007). Espejos en abismo. Estudios sobre la intertextualidad en la plástica. Guadalajara, México: Universidad de Guadalajara. 